\title{
Robust Stabilization of Discrete Model-Reference Control Systems on Integer Grid Coordinates
}

\author{
Yoshifumi Okuyama
}

\begin{abstract}
This paper describes a robust stabilization problem of discrete model-reference control systems on integer grid coordinates. Currently, all feedback control systems are realized using discretized signals. However, the analysis and design of discrete-time and discrete-value (point-to-point) control systems has not been established. In this paper, the robust stability of that type of discretized control systems is examined in a frequency domain. A robust stability condition for such discrete control systems with multi-nonlinearity is derived by applying Ostrowski's M-matrix. Using these results, the stabilization and design of model-reference control systems is performed. It can be seen that the model-reference control using a secondorder lag system is equivalently transformed into a traditional PID control scheme. Numerical examples for discrete modelreference control are provided to verify the design method.
\end{abstract}

\section{INTRODUCTION}

At present, all feedback control systems are realized using discretized (discrete-time and discrete-value) signals. However, the analysis and design of discretized (point-to-point) control systems has not been entirely elucidated. An attempt to elucidate the quantized control systems was presented first in a paper [1]. Since then, the problem of mitigating the quantization effects in quantized control systems has been studied [2], [3], [4], [5]. However, few results have been obtained for the analysis and design of that type of nonlinear discrete-time control systems [6], [7].

In our previous papers [8], [9], [10], the robust stabilization of nonlinear discrete-time and discrete-value (discretized) control systems was examined in a frequency domain. In the study, a traditional (discrete-time) PID control scheme was used in the controller design. Moreover, based on model-reference feedback structure a design method of such discretized control systems was presented in [11]. In the study, it was shown that the model-reference feedback using a second-order lag system is equivalently transformed into a PID control scheme. In this paper, the robust stability of that type of discretized control systems is examined in a frequency domain. A robust stability condition for such discrete control systems with multi-nonlinearity is derived by applying Ostrowski's M-matrix [12]. Using these results, the stabilization and design of model-reference control systems is performed.

\section{Discretized Model ReFEREnCE FEedBACK}

Model-reference feedback structure for a robust control system was once proposed by the authors [13]. A discretetime version of the model reference feedback was presented

Yoshifumi Okuyama is a director and a professor emeritus at Humanitech Laboratory Co., Ltd., 115-7, Nakatsuura, Hachiman-cho, Tokushima, 7708072, Japan oku@humanitech-lab.jp

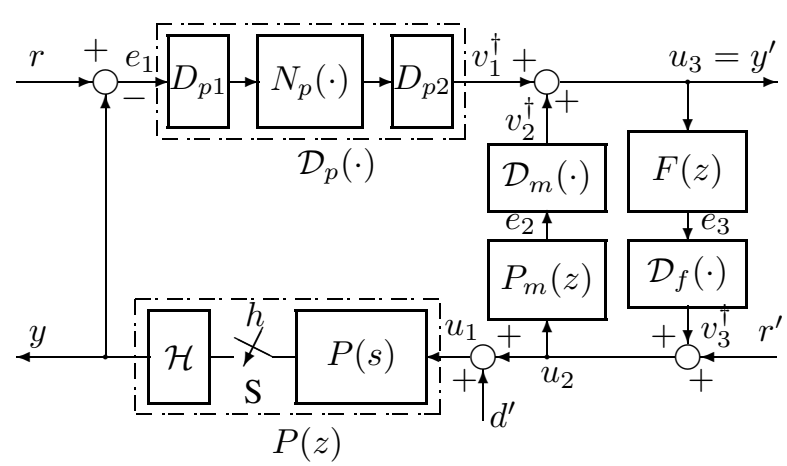

Fig. 1. Discretized model-reference feedback control system.

in [14]. In this paper, a discretized model reference control system as shown in Fig. 1 is examined. Here, $P(s)$ is a continuous-time and continuous-value plant, and $P(z)$ is the $z$-transform of $P(s)$ together with the zero-order hold $\mathcal{H}$. In addition, $N_{p}(\cdot), D_{p 1}$ and $D_{p 2}$ are a nonlinear continuous element and the input/output discretizing units which are performed in A/D (D/A) conversion. Moreover, $P_{m}(z)$ is the plant model and $F(z)$ is the feedback compensator. $\mathcal{D}_{m}(\cdot)$ and $\mathcal{D}_{f}(\cdot)$ are the nonlinear discretized elements of the model and the compensator, respectively.

In the figure, each symbol $e_{1}, v_{1}, \cdots$ indicates the sequence $e_{1}(k), v_{1}(k), \cdots(k=0,1,2, \cdots)$ in discrete time, but for continuous value. On the other hand, each symbol $e_{1}^{\dagger}$, $v_{1}^{\dagger}, \cdots$ indicates a discrete value that can be assigned to an integer number, e.g.,

$$
\begin{aligned}
& e_{1}^{\dagger} \in\{\cdots,-2 \gamma,-\gamma, 0, \gamma, 2 \gamma, \cdots\}, \\
& v_{1}^{\dagger} \in\{\cdots,-2 \gamma,-\gamma, 0, \gamma, 2 \gamma, \cdots\},
\end{aligned}
$$

where $\gamma$ is the resolution of each variable. An example of the discretization characteristics $D_{p 1}, D_{p 2}$, and the discretized characteristic $v_{1}^{\dagger}=\mathcal{D}_{p}\left(e_{1}\right)$ is depicted as shown in Fig. 2. Here, without loss of generality, the resolutions in $D_{p 1}$ and $D_{p 2}$ are assumed to be $\gamma=1.0$, and the continous nonlinear curve $N_{p}(\cdot)$ is chosen as a sigmoid function.

\section{MOdEL System AND BilineAR TRANSFORMATION}

In this study, the model of plant, $P_{m}(z)$, is assumed to be a second-order lag system, e.g.,

$$
P_{m}(z)=\hat{P}_{m}(\delta)=\frac{1}{1+C_{1} \delta+C_{2} \delta^{2}},
$$

where $\delta$ is the following bilinear transformation:

$$
\delta=\frac{2}{h} \cdot \frac{z-1}{z+1} \quad(h \text { : sampling period }) .
$$

Here, $C_{1}$ and $C_{2}$ are the design parameters of the model system. 

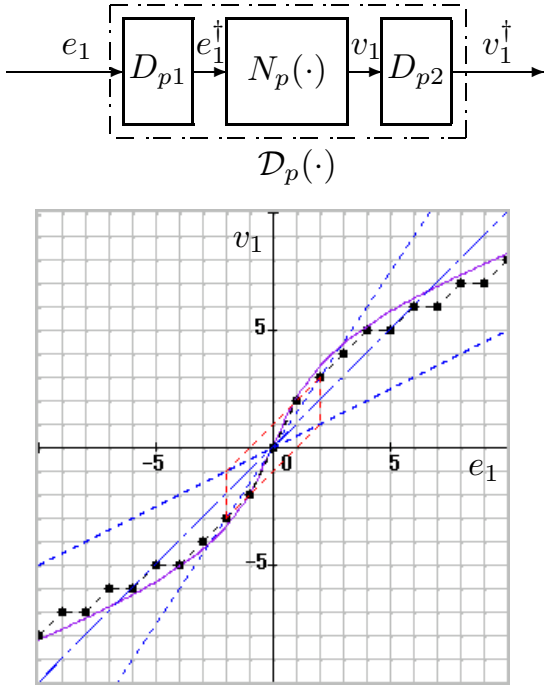

Fig. 2. Discretization for a nonlinear characteristic.

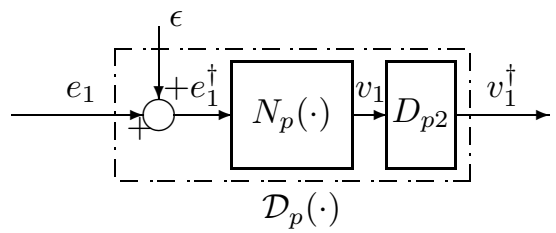

Fig. 3. Equivalent expression $\left(\epsilon=e_{1}^{\dagger}-e_{1}\right.$ : a sawtooth signal).

The $z$-transform expression of (2) is given by

$P_{m}(z)=\frac{h^{2}(z+1)^{2}}{h^{2}(z+1)^{2}+2 C_{1} h(z+1)(z-1)+4 C_{2}(z-1)^{2}}$.

Obviously, $\delta$ approaches Laplace transform variable $s$, when the sampling period is $h \rightarrow 0$.

The operator $\delta$ has the following properties:

1) Since $\delta^{-1}=\frac{h}{2} \cdot \frac{1+z^{-1}}{1-z^{-1}}$, the relationship between the input and output sequences, $x(k), y(k),(k=$ $0,1,2, \cdots)$ can be written as:

$$
y(k)=y(k-1)+\frac{h}{2}(x(k)+x(k-1)) .
$$

This transformation corresponds to a trapezoidal summation (integration).

2) On the other hand, with respect to operator $\delta$, the following relation is obtained:

$$
y(k)=-y(k-1)+\frac{2}{h}(x(k)-x(k-1)) .
$$

In the frequency domain, $\delta$ can be expressed by pure imaginary number as follows:

$$
\delta\left(\mathrm{e}^{j \omega h}\right)=j \Omega(\omega)=j \frac{2}{h} \tan \left(\frac{\omega h}{2}\right), \quad j=\sqrt{-1},
$$

where $\Omega$ is a distorted frequency of $\omega$.

In this paper, hereafter, variables and transfer functions of $z$ will be expressed by $\delta$ based on

$$
z=\frac{1+(1 / 2) h \delta}{1-(1 / 2) h \delta}
$$

\section{Discretized Nonlinear CHARACTERISTICS AND INEQUALITY CONDITIONS}

The input/output discretization processes and the discretized nonlinear characteristics are illustrated as shown in Fig. 2. The nonlinear characteristics can be partitioned as follows:

$$
\begin{aligned}
& v_{1}^{\dagger}=\mathcal{D}_{p}\left(e_{1}^{\dagger}\right)=K e_{1}^{\dagger}+g_{1}\left(e_{1}^{\dagger}\right), \quad 0<K<\infty \\
& \left|w_{1}^{\dagger}\right|=\left|g_{1}\left(e_{1}^{\dagger}\right)\right|<\infty, \quad \text { for }\left|e_{1}^{\dagger}\right|<\varepsilon_{1} \\
& \left|w_{1}^{\dagger}\right|=\left|g_{1}\left(e_{1}^{\dagger}\right)\right| \leq \beta_{1}\left|e_{1}^{\dagger}\right|, \quad \text { for }\left|e_{1}^{\dagger}\right| \geq \varepsilon_{1} .
\end{aligned}
$$

Since the input-side discretization is equivalently represented as shown in Fig. 3, the input signal is considered at an integer, $e_{1}=e_{1}^{\dagger}$ (Here, $w_{1}^{\dagger}$ is not always an integer though $\dagger$ is attached to the symbol.)

In these inequalities, when analyzing the robust stability in a global sense, it is sufficient to consider the nonlinear term (10), because the nonlinear term (9) can be treated as a disturbance signal. In this study, since the nonlinear characteristic (8) is assumed to exist in the first and the third quadrant, the sector parameter $\beta_{1}$ should be considered in $0 \leq \beta_{1} \leq K$.

In regard to the model system, the discretized nonlinear characteristic can be expressed as:

$$
\begin{aligned}
& v_{2}^{\dagger}=\mathcal{D}_{m}\left(e_{2}^{\dagger}\right)=K_{m} e_{2}^{\dagger}+g_{2}\left(e_{2}^{\dagger}\right), \quad 0<K_{m}<\infty \\
& \left|w_{2}^{\dagger}\right|=\left|g_{2}\left(e_{2}^{\dagger}\right)\right|<\infty, \text { for }\left|e_{2}^{\dagger}\right|<\varepsilon_{2}, \\
& \left|w_{2}^{\dagger}\right|=\left|g_{2}\left(e_{2}^{\dagger}\right)\right| \leq \beta_{2}\left|e_{2}^{\dagger}\right|, \text { for }\left|e_{2}^{\dagger}\right| \geq \varepsilon_{2},
\end{aligned}
$$

and $0 \leq \beta_{2} \leq K_{m}$. Moreover, in regard to the feedback compensator, the following expression can be given:

$$
\begin{aligned}
& v_{3}^{\dagger}=\mathcal{D}_{f}\left(e_{3}^{\dagger}\right)=K_{f} e_{3}^{\dagger}+g_{3}\left(e_{3}^{\dagger}\right), \quad 0<K_{f}<\infty \\
& \left|w_{3}^{\dagger}\right|=\left|g_{3}\left(e_{3}^{\dagger}\right)\right|<\infty, \quad \text { for }\left|e_{3}^{\dagger}\right|<\varepsilon_{3} \\
& \left|w_{3}^{\dagger}\right|=\left|g_{3}\left(e_{3}^{\dagger}\right)\right| \leq \beta_{3}\left|e_{3}^{\dagger}\right|, \quad \text { for }\left|e_{3}^{\dagger}\right| \geq \varepsilon_{3}
\end{aligned}
$$

and $0 \leq \beta_{3} \leq K_{f}$.

Therefore, the robust stability of discretized systems as described above is analyzed based on the inner-product and norm analysis in the $\ell_{2}$ space. With respect to (10), the following new nonlinear function can be defined ${ }^{1}$ :

$$
f_{i}\left(e_{i}\right):=g_{i}\left(e_{i}\right)+\beta_{i} \cdot e_{i}, \quad i=1,2,3 .
$$

When considering the discretized output of the nonlinear characteristic, $w_{i}^{\dagger}=g_{i}\left(e_{i}^{\dagger}\right)$, the following expression is given:

$$
f_{i}\left(e_{i}^{\dagger}(k)\right)=w_{i}^{\dagger}(k)+\beta_{i} \cdot e_{i}^{\dagger}(k) .
$$

From inequality (10), it can be seen that (18) belongs to the first and third quadrants.

For the neutral points of $e_{i}^{\dagger}(k)$ and $w_{i}^{\dagger}(k)$, the following expression can be given from (18):

$$
\frac{1}{2}\left(f_{i}\left(e_{i}^{\dagger}(k)\right)+f_{i}\left(e_{i}^{\dagger}(k-1)\right)\right)=\bar{w}_{i}^{\dagger}(k)+\beta_{i} \cdot \bar{e}_{i}^{\dagger}(k),
$$

\footnotetext{
${ }^{1}$ Hereafter, only $i=1$ is considered.
} 
where

$$
\bar{w}_{i}^{\dagger}(k)=\frac{w_{i}^{\dagger}(k)+w_{i}^{\dagger}(k-1)}{2}, \bar{e}_{i}^{\dagger}(k)=\frac{e_{i}^{\dagger}(k)+e_{i}^{\dagger}(k-1)}{2} .
$$

Then, the trapezoidal area of one-step transition on integer grid coordinates, $f_{i}(e)$, is written as follows:

$$
\begin{aligned}
\tau_{i}(k) & :=\frac{1}{2}\left(f_{i}\left(e^{\dagger}(k)\right)+f_{i}\left(e_{i}^{\dagger}(k-1)\right)\right) \Delta e_{i}^{\dagger}(k) \\
& =\left(\bar{w}_{i}^{\dagger}(k)+\beta_{i} \bar{e}_{i}^{\dagger}(k)\right) \Delta e_{i}^{\dagger}(k),
\end{aligned}
$$

Here, $\Delta e_{i}^{\dagger}(k)$ is the backward difference of sequence $e_{i}^{\dagger}(k)$.

$$
\Delta e_{i}^{\dagger}(k)=e_{i}^{\dagger}(k)-e_{i}^{\dagger}(k-1) .
$$

Since $f\left(e_{i}^{\dagger}(k)\right)$ belongs to the first and third quadrants, the area of each trapezoid $\tau_{i}(k)$ is non-negative when $e_{i}(k)$ increases (decreases) in the first (third) quadrant. On the other hand, the trapezoidal area $\tau_{i}(k)$ is non-positive when $e_{i}(k)$ decreases (increases) in the first (third) quadrant.

In our study, the following assumption is provided with respect to the discretized responses on the integer grid coordinates.

[Assumption] The absolute value of the backward difference of sequence $e(k)$ does not exceed $\gamma$, i.e.,

$$
\left|\Delta e_{i}(k)\right|=\left|e_{i}(k)-e_{i}(k-1)\right| \leq \gamma .
$$

If condition (22) is satisfied, $\Delta e^{\dagger}(k)$ is exactly $\pm \gamma$ or 0 because of the discretization. That is, the absolute value of the backward difference can be given as

$$
\left|\Delta e_{i}^{\dagger}(k)\right|=\left|e_{i}^{\dagger}(k)-e_{i}^{\dagger}(k-1)\right|=\gamma \text { or } 0 .
$$

This assumption states that each point of the response traces on adjacent points in the integer grid coordinates.

Consider the following sum of trapezoidal areas:

$$
\sigma_{i}(p)=\sum_{k=1}^{p} \tau_{i}(k)
$$

If the above assumption is satisfied with respect to the discretization of the control system, the sum of trapezoidal areas, $\sigma(p)$, becomes non-negative for any $p$. Since the discretized output traces the same points on the stepwise nonlinear characteristic, the sum of trapezoidal areas is canceled when $e_{i}(k)$ (and $e_{i}^{\dagger}(k)$ decreases (increases) from a certain point $\left(e_{i}^{\dagger}(k), f_{i}\left(e_{i}^{\dagger}(k)\right)\right)$ in the first (third) quadrant. (Here, without loss of generality, the response of discretized point $\left(e_{i}^{\dagger}(k), f_{i}\left(e_{i}^{\dagger}(k)\right)\right)$ is assumed to commence at the origin.)

From equation (21), the sum of trapezoidal areas can be expressed as follows:

$$
\begin{aligned}
\sigma_{i}(p) & :=\frac{1}{2} \sum_{k=1}^{p}\left(f_{i}\left(e_{i}^{\dagger}(k)\right)+f_{i}\left(e_{i}^{\dagger}(k-1)\right)\right) \Delta e_{i}^{\dagger}(k) \\
& =\left\langle\bar{w}_{i}^{\dagger}(k)+\beta_{i} \bar{e}_{i}^{\dagger}(k), \Delta \bar{e}_{i}^{\dagger}(k)\right\rangle_{p}
\end{aligned}
$$

Here, $\langle\cdot, \cdot\rangle_{p}$ denotes the inner product in the $\ell_{2}$ space,

$$
\langle x(k), y(k)\rangle_{p}=\sum_{k=1}^{p} x(k) y(k) .
$$

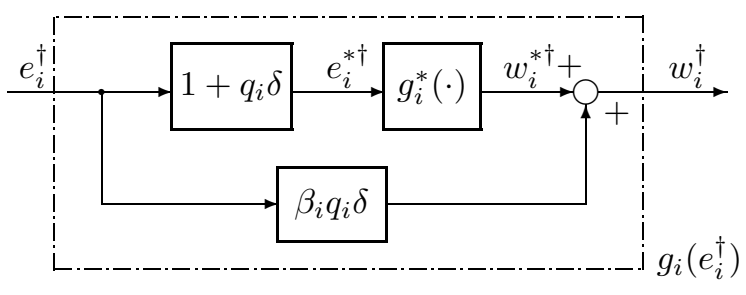

Fig. 4. Nonlinear subsystem $(i=1,2,3)$.

In order to derive the robust stability condition, the following new sequences are considered here:

$$
\begin{aligned}
& \bar{e}_{i}^{* \dagger}(k)=\bar{e}_{i}^{\dagger}(k)+q_{i} \cdot \frac{\Delta e_{i}^{\dagger}(k)}{h}, \\
& \bar{w}_{i}^{* \dagger}(k)=\bar{w}_{i}^{\dagger}(k)-\beta_{i} q_{i} \cdot \frac{\Delta e_{i}^{\dagger}(k)}{h},
\end{aligned}
$$

where $q_{i}$ is a non-negative number. The relationship between equations (25) and (26) is as shown in Fig. 4.

Based on these sequences, the following lemma is given [15], [16]:

[Lemma] If the following inequality is satisfied with respect to the inner product of the neutral points of (18) and the backward difference:

$$
\left\langle\bar{w}_{i}^{\dagger}(k)+\beta_{i} \bar{e}_{i}^{\dagger}(k), \Delta e_{i}^{\dagger}(k)\right\rangle_{p} \geq 0,
$$

the following inequality can be obtained:

$$
\left\|\bar{w}_{i}^{* \dagger}(k)\right\|_{2, p} \leq \beta_{i}\left\|\bar{e}_{i}^{* \dagger}(k)\right\|_{2, p} \leq \beta_{i}\left\|\bar{e}_{i}^{*}(k)\right\|_{2, p}
$$

for any $q_{i} \geq 0$ and $p \rightarrow \infty$. Here, $\|\cdot\|_{2, p}$ denotes the Euclidean norm, which can be defined by

$$
\|x(k)\|_{2, p}:=\left(\sum_{k=1}^{p}|x(k)|^{2}\right)^{1 / 2}
$$

(Proof) The following equation is obtained from (25) and (26):

$$
\begin{aligned}
& \beta_{i}^{2}\left\|\bar{e}_{i}^{* \dagger}(k)\right\|_{2, p}^{2}-\left\|\bar{w}_{i}^{* \dagger}(k)\right\|_{2, p}^{2} \\
& \quad=\beta_{i}^{2}\left\|\bar{e}_{i}^{\dagger}(k)\right\|_{2, p}^{2}-\left\|\bar{w}_{i}^{\dagger}(k)\right\|_{2, p}^{2} \\
& \quad+\frac{2 \beta_{i} q_{i}}{h} \cdot\left\langle\bar{w}_{i}^{\dagger}(k)+\beta_{i} \bar{e}_{i}^{\dagger}(k), \Delta e_{i}^{\dagger}(k)\right\rangle_{p} .
\end{aligned}
$$

From (10), (13), and (16), the following holds:

$$
\left\|\bar{w}_{i}^{\dagger}(k)\right\|_{2, p} \leq \beta_{i}\left\|\bar{e}_{i}^{\dagger}(k)\right\|_{2, p} .
$$

Thus, the following inequality can be obtained from (27)

$$
\left\|\bar{w}_{i}^{* \dagger}(k)\right\|_{2, p} \leq \beta_{i}\left\|\bar{e}_{i}^{* \dagger}(k)\right\|_{2, p} .
$$

Since $\left\|\bar{e}_{i}^{\dagger}(k)\right\|_{2, p} \leq\left\|\bar{e}_{i}(k)\right\|_{2, p}$ for $p \rightarrow \infty$, the following inequality holds in the frequency domain based on Parseval's formula ${ }^{2}$ :

$$
\begin{aligned}
& \left\|\bar{e}_{i}^{* \dagger}(\delta)\right\|_{2}=\left|1+q_{i} \delta\right| \cdot\left\|\bar{e}_{i}^{\dagger}(\delta)\right\|_{2} \\
& \quad \leq\left|1+q_{i} \delta\right| \cdot\left\|\bar{e}_{i}(\delta)\right\|_{2}=\left\|\bar{e}_{i}^{*}(\delta)\right\|_{2} .
\end{aligned}
$$

Then, $\left\|\bar{e}_{i}^{* \dagger}(k)\right\|_{2, p} \leq\left\|\bar{e}_{i}^{*}(k)\right\|_{2, p}$, and thus the right-side of inequality (28) is satisfied.

\footnotetext{
${ }^{2}$ Hereafter, $\delta$ is considered $j \Omega(\omega)$ as shown in (7)
} 


\section{VECTOR-MATRIX EXPRESSION FOR MODEL-REFERENCE CONTROL}

The model-reference control system as shown in Fig. 1 is given by the following vector-matrix expression:

$$
\left[\begin{array}{l}
e_{1}(\delta) \\
e_{2}(\delta) \\
e_{3}(\delta)
\end{array}\right]=\left[\begin{array}{l}
1 \\
0 \\
0
\end{array}\right] r(\delta)+\left[\begin{array}{ccc}
-P(\delta) & 0 & 0 \\
0 & P_{m}(\delta) & 0 \\
0 & 0 & F(\delta)
\end{array}\right]\left[\begin{array}{l}
u_{1}(\delta) \\
u_{2}(\delta) \\
u_{3}(\delta)
\end{array}\right],
$$

where control inputs $u_{1}, u_{2}$, and $u_{3}$ are given by

$$
\left[\begin{array}{l}
u_{1}(\delta) \\
u_{2}(\delta) \\
u_{3}(\delta)
\end{array}\right]=\left[\begin{array}{c}
u_{2}(\delta) \\
v_{3}^{\dagger}(\delta) \\
v_{1}^{\dagger}(\delta)+v_{2}^{\dagger}(\delta)
\end{array}\right]+\left[\begin{array}{c}
d^{\prime}(\delta) \\
r^{\prime}(\delta) \\
0
\end{array}\right]
$$

As shown in Fig. 4, the nonlinear parts (point-to-point characteristics) of the system can be written as follows ${ }^{3}$ :

$$
\left[\begin{array}{l}
v_{1}^{\dagger} \\
v_{2}^{\dagger} \\
v_{3}^{\dagger}
\end{array}\right]=\left[\begin{array}{ccc}
\Gamma_{1}(\delta) & 0 & 0 \\
0 & \Gamma_{2}(\delta) & 0 \\
0 & 0 & \Gamma_{3}(\delta)
\end{array}\right]\left[\begin{array}{c}
e_{1}^{\dagger} \\
e_{2}^{\dagger} \\
e_{3}^{\dagger}
\end{array}\right]+\left[\begin{array}{c}
w_{1}^{* \dagger} \\
w_{2}^{* \dagger} \\
w_{3}^{* \dagger}
\end{array}\right],
$$

where $\Gamma_{1}(\delta)=K+\beta_{1} q_{1} \delta, \Gamma_{2}(\delta)=K_{m}+\beta_{2} q_{2} \delta, \Gamma_{3}(\delta)=$ $K_{f}+\beta_{3} q_{3} \delta$.

If exogenous inputs are $r^{\prime}=d^{\prime}=0, u_{1}$ is equal to $u_{2}$. Moreover, since $e_{i}^{\dagger}=e_{i}-d_{i}(i=1,2,3)$, the following closed-loop system equation can be given:

$$
\begin{aligned}
& {\left[\begin{array}{l}
e_{1}^{\dagger} \\
e_{2}^{\dagger} \\
e_{3}^{\dagger}
\end{array}\right]=\left[\begin{array}{c}
d_{1}+r \\
d_{2} \\
d_{3}
\end{array}\right]+\left[\begin{array}{cc}
-P & 0 \\
P_{m} & 0 \\
0 & F
\end{array}\right]\left[\begin{array}{l}
u_{1} \\
u_{3}
\end{array}\right]} \\
& =\left[\begin{array}{c}
d_{1}+r \\
d_{2} \\
d_{3}
\end{array}\right]+\left[\begin{array}{cc}
-P & 0 \\
P_{m} & 0 \\
0 & F
\end{array}\right]\left[\begin{array}{ccc}
0 & 0 & \Gamma_{3} \\
\Gamma_{1} & \Gamma_{2} & 0
\end{array}\right]\left[\begin{array}{l}
e_{1}^{\dagger} \\
e_{2}^{\dagger} \\
e_{3}^{\dagger}
\end{array}\right] \\
& +\left[\begin{array}{cc}
-P & 0 \\
P_{m} & 0 \\
0 & F
\end{array}\right]\left[\begin{array}{lll}
0 & 0 & 1 \\
1 & 1 & 0
\end{array}\right]\left[\begin{array}{c}
w_{1}^{* \dagger} \\
w_{2}^{* \dagger} \\
w_{3}^{* \dagger}
\end{array}\right],
\end{aligned}
$$

where $d_{i}\left(\left|d_{i}\right| \leq \gamma\right)$ are discretized/quantized errors.

As for the neutral points defined in (20), the following expression can be given:

$$
\begin{gathered}
{\left[\begin{array}{ccc}
1 & 0 & \Gamma_{3} P \\
0 & 1 & -\Gamma_{3} P_{m} \\
-\Gamma_{1} F & -\Gamma_{2} F & 1
\end{array}\right]\left[\begin{array}{l}
\bar{e}_{1}^{\dagger} \\
\bar{e}_{2}^{\dagger} \\
\bar{e}_{3}^{\dagger}
\end{array}\right]} \\
=\left[\begin{array}{c}
\bar{d}_{1}+\bar{r} \\
\bar{d}_{2} \\
\bar{d}_{3}
\end{array}\right]\left[\begin{array}{ccc}
0 & 0 & -P \\
0 & 0 & P_{m} \\
F & F & 0
\end{array}\right]\left[\begin{array}{l}
\bar{w}_{1}^{* \dagger} \\
\bar{w}_{2}^{* \dagger} \\
\bar{w}_{3}^{* \dagger}
\end{array}\right] .
\end{gathered}
$$

Inverse matrix of the left side of the equation is written as follows:

$$
\begin{aligned}
& {\left[\begin{array}{ccc}
1 & 0 & \Gamma_{3} P \\
0 & 1 & -\Gamma_{3} P_{m} \\
-\Gamma_{1} F & -\Gamma_{2} F & 1
\end{array}\right]^{-1}=\frac{1}{1+\left(\Gamma_{1} P-\Gamma_{2} P_{m}\right) \Gamma_{3} F}} \\
& \cdot\left[\begin{array}{ccc}
1-\Gamma_{2} \Gamma_{3} P_{m} F & -\Gamma_{2} \Gamma_{3} P F & -\Gamma_{3} P \\
\Gamma_{1} \Gamma_{3} P_{m} F & 1+\Gamma_{1} \Gamma_{3} P F & \Gamma_{3} P_{m} \\
\Gamma_{1} F & \Gamma_{2} F & 1
\end{array}\right] \\
& =\left[\begin{array}{lll}
\Psi_{11} & \Psi_{12} & \Psi_{13} \\
\Psi_{21} & \Psi_{22} & \Psi_{23} \\
\Psi_{31} & \Psi_{32} & \Psi_{33}
\end{array}\right] .
\end{aligned}
$$

${ }^{3}$ Hereafter, no confusion is possible, e.g., $e_{1}(\delta)$ and $P(\delta)$ will be abbreviated to simply $e_{1}$ and $P$.
Hereafter, in order to simplify the equation, the following symbols will be used:

$$
\Lambda_{i}(\delta)=1+q_{i} \delta, \quad i=1,2,3 .
$$

Thus,

$$
\left[\begin{array}{l}
\bar{e}_{1}^{* \dagger}(\delta) \\
\bar{e}_{2}^{* \dagger}(\delta) \\
\bar{e}_{3}^{* \dagger}(\delta)
\end{array}\right]=\left[\begin{array}{ccc}
\Lambda_{1}(\delta) & 0 & 0 \\
0 & \Lambda_{2}(\delta) & 0 \\
0 & 0 & \Lambda_{3}(\delta)
\end{array}\right]\left[\begin{array}{c}
\bar{e}_{1}^{\dagger}(\delta) \\
\bar{e}_{2}^{\dagger}(\delta) \\
\bar{e}_{3}^{\dagger}(\delta)
\end{array}\right]
$$

Hence, the vector-matrix expression of the control system is written by functions of $\delta$ as follows:

$$
\begin{aligned}
& {\left[\begin{array}{c}
\bar{e}_{1}^{* \dagger} \\
\bar{e}_{2}^{* \dagger} \\
\bar{e}_{3}^{* \dagger}
\end{array}\right]=\left[\begin{array}{ccc}
\Lambda_{1} & 0 & 0 \\
0 & \Lambda_{2} & 0 \\
0 & 0 & \Lambda_{3}
\end{array}\right]\left[\begin{array}{lll}
\Psi_{11} & \Psi_{12} & \Psi_{13} \\
\Psi_{21} & \Psi_{22} & \Psi_{23} \\
\Psi_{31} & \Psi_{32} & \Psi_{33}
\end{array}\right]\left[\begin{array}{c}
\bar{d}_{1}+\bar{r} \\
\bar{d}_{2} \\
\bar{d}_{3}
\end{array}\right]} \\
& +\left[\begin{array}{ccc}
\Lambda_{1} & 0 & 0 \\
0 & \Lambda_{2} & 0 \\
0 & 0 & \Lambda_{3}
\end{array}\right]\left[\begin{array}{lll}
\Psi_{11} & \Psi_{12} & \Psi_{13} \\
\Psi_{21} & \Psi_{22} & \Psi_{23} \\
\Psi_{31} & \Psi_{32} & \Psi_{33}
\end{array}\right]\left[\begin{array}{ccc}
0 & 0 & -P \\
0 & 0 & P_{m} \\
F & F & 0
\end{array}\right]\left[\begin{array}{c}
\bar{w}_{1}^{* \dagger} \\
\bar{w}_{2}^{* \dagger} \\
\bar{w}_{3}^{* \dagger}
\end{array}\right] \\
& =\left[\begin{array}{lll}
\Lambda_{1} \Psi_{11} & \Lambda_{1} \Psi_{12} & \Lambda_{1} \Psi_{13} \\
\Lambda_{2} \Psi_{21} & \Lambda_{2} \Psi_{22} & \Lambda_{2} \Psi_{23} \\
\Lambda_{3} \Psi_{31} & \Lambda_{3} \Psi_{32} & \Lambda_{3} \Psi_{33}
\end{array}\right]\left[\begin{array}{c}
\bar{d}_{1}+\bar{r} \\
\bar{d}_{2} \\
\bar{d}_{3}
\end{array}\right] \\
& +\left[\begin{array}{lll}
\Lambda_{1} \Psi_{13} F & \Lambda_{1} \Psi_{13} F & -\Lambda_{1}\left(\Psi_{11} P-\Psi_{12} P_{m}\right) \\
\Lambda_{2} \Psi_{23} F & \Lambda_{2} \Psi_{23} F & -\Lambda_{2}\left(\Psi_{21} P-\Psi_{22} P_{m}\right) \\
\Lambda_{3} \Psi_{33} F & \Lambda_{3} \Psi_{33} F & -\Lambda_{3}\left(\Psi_{31} P-\Psi_{32} P_{m}\right)
\end{array}\right]\left[\begin{array}{c}
\bar{w}_{1}^{* \dagger} \\
\bar{w}_{2}^{* \dagger} \\
\bar{w}_{3}^{* \dagger}
\end{array}\right] .
\end{aligned}
$$

In regard to to each norm of the equation, the following inequality is obtained:

$\left[\begin{array}{c}\left\|\bar{e}_{1}^{* \dagger}\right\|_{2} \\ \left\|\left.\right|_{2} ^{* \dagger}\right\|_{2} \\ \left\|\bar{e}_{3}^{* \dagger}\right\|_{2}\end{array}\right] \leqq\left[\begin{array}{lll}\left|\Lambda_{1} \Psi_{11}\right| & \left|\Lambda_{1} \Psi_{12}\right| & \left|\Lambda_{1} \Psi_{13}\right| \\ \left|\Lambda_{2} \Psi_{21}\right| & \left|\Lambda_{2} \Psi_{22}\right| & \left|\Lambda_{2} \Psi_{23}\right| \\ \left|\Lambda_{3} \Psi_{31}\right| & \left|\Lambda_{3} \Psi_{32}\right| & \left|\Lambda_{3} \Psi_{33}\right|\end{array}\right]\left[\begin{array}{c}\left\|\bar{d}_{1}\right\|_{2}+\|\bar{r}\|_{2} \\ \left\|\bar{d}_{2}\right\|_{2} \\ \left\|\bar{d}_{3}\right\|_{2}\end{array}\right]$
$\left[\begin{array}{lll}\left|\Lambda_{1} \Psi_{13} F\right| & \left|\Lambda_{1} \Psi_{13} F\right| & \left|\Lambda_{1}\left(\Psi_{11} P-\Psi_{12} P_{m}\right)\right| \\ \left|\Lambda_{2} \Psi_{23} F\right| & \left|\Lambda_{2} \Psi_{23} F\right| & \left|\Lambda_{2}\left(\Psi_{21} P-\Psi_{22} P_{m}\right)\right| \\ \left|\Lambda_{3} \Psi_{33} F\right| & \left|\Lambda_{3} \Psi_{33} F\right| & \left|\Lambda_{3}\left(\Psi_{31} P-\Psi_{32} P_{m}\right)\right|\end{array}\right]\left[\begin{array}{l}\left\|\bar{w}_{1}^{* \dagger}\right\|_{2} \\ \left\|\bar{w}_{2}^{* \dagger}\right\|_{2} \\ \left\|\bar{w}_{3}^{* \dagger}\right\|_{2}\end{array}\right]$.

Here, symbol $\leqq$ denotes a set of inequalities for each element.

\section{ROBUST STABILITY CONDITION FOR MULTI-NONLINEARITY SYSTEMS}

By using the result of Lemma, the following inequality is derived:

$\left[\begin{array}{ccc}1-\beta_{1}\left|\Lambda_{1} \Psi_{13} F\right| & -\beta_{2}\left|\Lambda_{1} \Psi_{13} F\right| & -\beta_{3}\left|\Lambda_{1}\left(\Psi_{11} P-\Psi_{12} P_{m}\right)\right| \\ -\beta_{1}\left|\Lambda_{2} \Psi_{23} F\right| & 1-\beta_{2}\left|\Lambda_{2} \Psi_{23} F\right| & -\beta_{3}\left|\Lambda_{2}\left(\Psi_{21} P-\Psi_{22} P_{m}\right)\right| \\ -\beta_{1}\left|\Lambda_{3} \Psi_{33} F\right| & -\beta_{2}\left|\Lambda_{3} \Psi_{33} F\right| & 1-\beta_{3}\left|\Lambda_{3}\left(\Psi_{31} P-\Psi_{32} P_{m}\right)\right|\end{array}\right]$
$\cdot\left[\begin{array}{c}\left\|\bar{e}_{1}^{* \dagger}\right\|_{2} \\ \left\|\bar{e}_{2}^{* \dagger}\right\|_{2} \\ \left\|\bar{e}_{3}^{* \dagger}\right\|_{2}\end{array}\right] \leqq\left[\begin{array}{ccc}\left|\Lambda_{1} \Psi_{11}\right| & \left|\Lambda_{1} \Psi_{12}\right| & \left|\Lambda_{1} \Psi_{13}\right| \\ \left|\Lambda_{2} \Psi_{21}\right| & \left|\Lambda_{2} \Psi_{22}\right| & \left|\Lambda_{2} \Psi_{23}\right| \\ \left|\Lambda_{3} \Psi_{31}\right| & \left|\Lambda_{3} \Psi_{32}\right| & \left|\Lambda_{3} \Psi_{33}\right|\end{array}\right]\left[\begin{array}{c}\left\|\bar{d}_{1}\right\|_{2}+\|\bar{r}\|_{2} \\ \left\|\bar{d}_{2}\right\|_{2} \\ \left\|\bar{d}_{3}\right\|_{2}\end{array}\right]$.

When the matrix of the left side of the above inequality was written by:

$$
\mathcal{A}=\left[\begin{array}{lll}
a_{11} & a_{12} & a_{13} \\
a_{21} & a_{22} & a_{23} \\
a_{31} & a_{32} & a_{33}
\end{array}\right]
$$

all non-diagonal elements are obviously non-positive. In addition, if all diagonal elements are positive and if all 
principal minors of all order are positive, the above matrix $\mathcal{A}$ is called an M-matrix [17].

[Theorem-1] If there exists a $q_{i} \geq 0$ in which matrix (36) becomes an Ostrowski's M-matrix, the discretized modelreference control system with sector nonlinearities (10), (13), and (16) is robust stable in an $\ell_{2}$ sense, when the linearized system with nominal gains $K, K_{m}$, and $K_{f}$ is asymptotically stable.

(Proof) By using (36), inequality (35) can be written as follows:

$$
\left[\begin{array}{lll}
a_{11} & a_{12} & a_{13} \\
a_{21} & a_{22} & a_{23} \\
a_{31} & a_{32} & a_{33}
\end{array}\right]\left[\begin{array}{l}
x_{1} \\
x_{2} \\
x_{3}
\end{array}\right] \leqq\left[\begin{array}{l}
y_{1} \\
y_{2} \\
y_{3}
\end{array}\right]
$$

Obviously, elements of these vectors in (37) are $x_{i} \geq 0$ and $y_{i} \geq 0$. Moreover, with respect to elements of the matrix $\mathcal{A}$, $a_{i j} \leq 0(i \neq j)$ can be recognized.

The above inequality can be rewritten as follows:

$$
\left[\begin{array}{ccc}
a_{11}^{(1)} & a_{12}^{(1)} & a_{13}^{(1)} \\
0 & a_{22}^{(2)} & a_{23}^{(2)} \\
0 & 0 & a_{33}^{(3)}
\end{array}\right]\left[\begin{array}{l}
x_{1}^{(1)} \\
x_{2}^{(1)} \\
x_{3}^{(1)}
\end{array}\right] \leqq\left[\begin{array}{l}
y_{1}^{(1)} \\
y_{2}^{(2)} \\
y_{3}^{(3)}
\end{array}\right]
$$

where

$$
a_{i j}^{(1)}=a_{i j}, \quad x_{j}^{(1)}=x_{j}, \quad y_{i}^{(1)}=y_{i}(i, j=1,2,3) .
$$

Furthermore,

$$
\left\{\begin{array}{l}
a_{22}^{(2)}=\frac{1}{a_{11}^{(1)}}\left|\begin{array}{ll}
a_{11}^{(1)} & a_{12}^{(1)} \\
a_{21}^{(1)} & a_{22}^{(1)}
\end{array}\right|, \quad a_{23}^{(2)}=\frac{1}{a_{11}^{(1)}}\left|\begin{array}{cc}
a_{11}^{(1)} & a_{13}^{(1)} \\
a_{21}^{(1)} & a_{23}^{(1)}
\end{array}\right|, \\
a_{32}^{(2)}=\frac{1}{a_{11}^{(1)}}\left|\begin{array}{cc}
a_{11}^{(1)} & a_{12}^{(1)} \\
a_{31}^{(1)} & a_{32}^{(1)}
\end{array}\right|, \quad a_{33}^{(2)}=\frac{1}{a_{11}^{(1)}}\left|\begin{array}{ll}
a_{11}^{(1)} & a_{13}^{(1)} \\
a_{31}^{(1)} & a_{33}^{(1)}
\end{array}\right|,
\end{array}\right.
$$

and

$$
a_{33}^{(3)}=\frac{1}{a_{22}^{(2)}}\left|\begin{array}{ll}
a_{22}^{(2)} & a_{23}^{(2)} \\
a_{32}^{(2)} & a_{33}^{(2)}
\end{array}\right|
$$

Therefore, the right side of (38) can be written as

$y_{1}^{(1)}=y_{1}, \quad y_{2}^{(2)}=y_{2}^{(1)}-\frac{a_{21}^{(1)}}{a_{11}^{(1)}} y_{1}^{(1)}, \quad y_{3}^{(3)}=y_{3}^{(2)}-\frac{a_{32}^{(2)}}{a_{22}^{(2)}} y_{2}^{(2)}$

provided $a_{11}^{(1)}>0$ and $a_{22}^{(2)}>0$. It can be seen that these values are non-negative and bounded if each norm of exogenous inputs is bounded (i.e., $\|\bar{r}\|_{2}<\infty,\left\|\bar{d}_{j}\right\|_{2}<\infty$, $(j=1,2,3)$. In addition, if $a_{33}^{(3)}>0$ is satisfied, $x_{3}^{(1)}<\infty$, $x_{2}^{(1)}<\infty$, and $x_{1}^{(1)}<\infty$ are obtained in reverse order.

Here, it should be noted that the above conditions $a_{11}^{(1)}>$ $0, a_{22}^{(2)}>0$, and $a_{33}^{(3)}>0$ are rewritten as follows:

$$
\begin{aligned}
& a_{11}^{(1)}=\Delta_{1}=a_{11}>0 \\
& a_{22}^{(2)}=\frac{\Delta_{2}}{\Delta_{1}}=\left|\begin{array}{ll}
a_{11} & a_{12} \\
a_{21} & a_{22}
\end{array}\right| / a_{11}>0 \\
& a_{33}^{(3)}=\frac{\Delta_{3}}{\Delta_{2}}=\left|\begin{array}{lll}
a_{11} & a_{12} & a_{13} \\
a_{21} & a_{22} & a_{23} \\
a_{31} & a_{32} & a_{33}
\end{array}\right| /\left|\begin{array}{ll}
a_{11} & a_{12} \\
a_{21} & a_{22}
\end{array}\right|>0 .
\end{aligned}
$$

The conditions say that all principal minors of matrix $\mathcal{A}$ are positive. That is, it means that the matrix becomes M-matrix.
(The derivation of the result in general form is written in Appendix.)

Thus, it can be proven that

$$
\left\|e_{i}^{*}\right\|<\infty \text { and }\left\|e_{i}\right\|<\infty, \quad i=1,2,3,
$$

when the nomianl control system with gains $K, K_{m}$, and $K_{f}$. The proof of Theorem-1 based on the concept of bounded-inputs and bounded-outputs (BIBO) stability of model-reference control systems is completed.

\section{RELATION TO PID CONTROL}

When the model and the feedback compensator are in higher resolution, in other word, $\beta_{2} \rightarrow 0$ and $\beta_{3} \rightarrow 0$, the model-reference control sytem can be transformed equivalently as shown in Fig. 5. Here, the equivalent controller and the pre-filter are given as

$$
\begin{aligned}
& C(\delta)=\frac{K_{f} F(\delta)}{1-K_{m} K_{f} P_{m}(\delta) F(\delta)}, \\
& D(\delta)=\frac{1}{K_{f} F(\delta)} .
\end{aligned}
$$

In the figure, $g_{1}(\cdot)$ can be replaced with the nonlinear subsytem as shown in Fig. 4. Therefore, the loop transfer function from $w_{1}^{*}$ to $e_{1}^{*}$ is given by

$$
H\left(\beta_{1}, q_{1}, \delta\right)=\frac{\left(1+q_{1} \delta\right) P(\delta) C(\delta)}{1+\left(K+\beta_{1} q_{1} \delta\right) P(\delta) C(\delta)} .
$$

Thus, the robust stability condition of the model-reference control will be rewritten as follows.

[Theorem-2] With respect to an arbitrary $q_{1} \geq 0$, the following inequality should be satisfied:

$$
\left|\frac{\left(1+q_{1} \delta\right) P(\delta) C(\delta)}{1+\left(K+\beta_{1} q_{1} \delta\right) P(\delta) C(\delta)}\right|<\frac{1}{\beta_{1}} .
$$

Arranging to an explicit form, inequality (42) will be equivalent to the theorem in the previous paper [11].

(Proof) When $\beta_{2}=\beta_{3}=0$ are assumed, the robust stability condition should be written from Theorem-1 as follows:

$$
\Delta_{1}=a_{11}=1-\beta_{1}\left|\Lambda_{1} \Psi_{13} F\right|>0 .
$$

From (31) and (32), inequality (43) can be expressed as

$$
1-\beta_{1}\left|\frac{-\left(1+q_{1} \delta\right) \Gamma_{3} P F}{1+\left(\Gamma_{1} P-\Gamma_{2} P_{m}\right) \Gamma_{3} F}\right|>0 \text {. }
$$

Since $\Gamma_{1}=K+\beta_{1} q_{1} \delta, \Gamma_{2}=K_{m}$, and $\Gamma_{3}=K_{f}$, inequality (44) is rewritten as follows:

$1-\beta_{1}\left|\frac{\left(1+q_{1} \delta\right) P(\delta) K_{f} F(\delta)}{1+\left[\left(K+\beta_{1} q_{1} \delta\right) P(\delta)-K_{m} P_{m}(\delta)\right] K_{f} F(\delta)}\right|>0$.

The above inequality is equivalent to (42).

As was descibed in section 3, when the model is assumed to be a second-order lag system, e.g.,

$$
K_{m} P_{m}(\delta)=\frac{K_{m}}{1+C_{1} \delta+C_{2} \delta^{2}},
$$




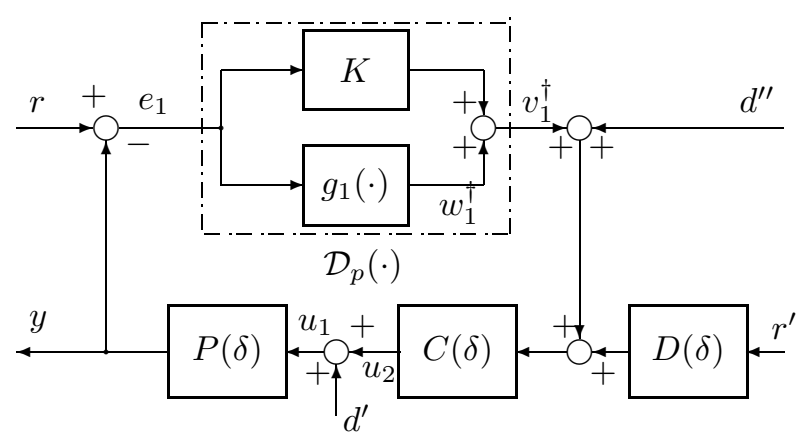

Fig. 5. Equivalent feedback control system.

the feedback compensator $K_{f} F(\delta)$ is defined in this study as follows:

$$
K_{f} F(\delta)=\frac{1+C_{1} \delta+C_{2} \delta^{2}}{K_{m}\left(1+c_{1} \delta+c_{2} \delta^{2}\right)},
$$

where $K_{m}$ will be substituted by $1 / K_{f}$.

In these cases, the equivalent controller and the pre-filter are given by

$$
\begin{aligned}
& C(\delta)=\frac{1+C_{1} \delta+C_{2} \delta^{2}}{K_{m}\left(c_{1} \delta+c_{2} \delta^{2}\right)}, \\
& D(\delta)=\frac{K_{m}\left(1+c_{1} \delta+c_{2} \delta^{2}\right)}{1+C_{1} \delta+C_{2} \delta^{2}} .
\end{aligned}
$$

Thus, the robust stability condition (45) can be rewritten as: $\left|\frac{\left(1+q_{1} \delta\right) P(\delta)\left(1+C_{1} \delta+C_{2} \delta^{2}\right)}{K_{m}\left(c_{1} \delta+c_{2} \delta^{2}\right)+\left(K+\beta_{1} q_{1} \delta\right) P(\delta)\left(1+C_{1} \delta+C_{2} \delta^{2}\right)}\right|<\frac{1}{\beta_{1}}$.

When $c_{2} \ll c_{1}$, the controller is approximately written as

$$
C(\delta)=\frac{1}{\kappa} \delta^{-1}+\frac{C_{1}}{\kappa}+\frac{C_{2}}{\kappa} \delta,
$$

where $\kappa=K_{m} c_{1}$. Obviously, (49) is a three-term controller based on the bilinear transformation expression, which corresponds to PID control for continuous-time systems.

When the model system is considered with time-delay,

$$
K_{m} P_{m}(\delta)=\frac{K_{m}}{1+C_{1} \delta+C_{2} \delta^{2}} \cdot \mathrm{e}^{-L_{m} s},
$$

the controller $C(\delta)$ is written as

$$
C(\delta)=\frac{1+C_{1} \delta+C_{2} \delta^{2}}{K_{m}\left(1+c_{1} \delta+c_{2} \delta^{2}-z^{-d_{m}}\right)},
$$

where $d_{m}$ is a time-delay that is written as $d_{m}=L_{m} / h$.

\section{NUMERICAL EXAMPLES}

[Example-1] Consider the following continuous plant:

$$
P(s)=\frac{K_{1}}{(s+0.1)(s+0.2)(s+0.5)},
$$

where the gain constant is $K_{1}=0.01$. The sampling period and the resolution value are assumed to be $h=1.0$ and $\gamma=1.0$. That is, the responses of the control systems trace on integer grid coordinates. The discretized nonlinear characteristic (discretized sigmoid, i.e. arc tangent) is as shown in Fig. 2.

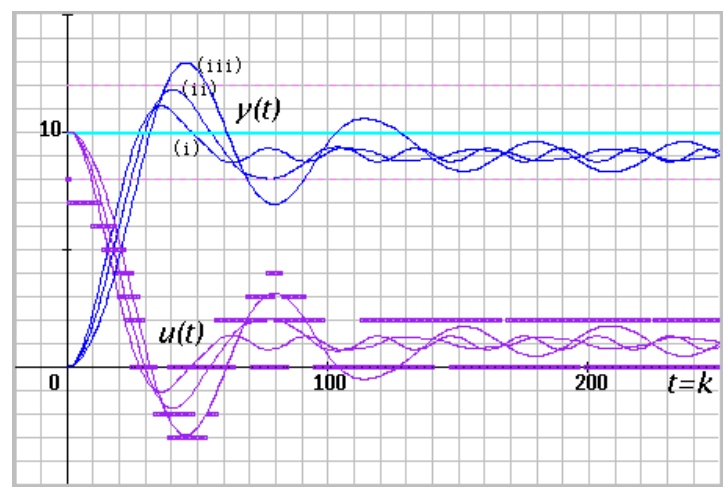

Fig. 6. Step responses for Exmaple-1 ((i) $C_{1}=8.0$, (ii) $C_{1}=5.0$, (iii) $\left.C_{1}=2.0\right)$.

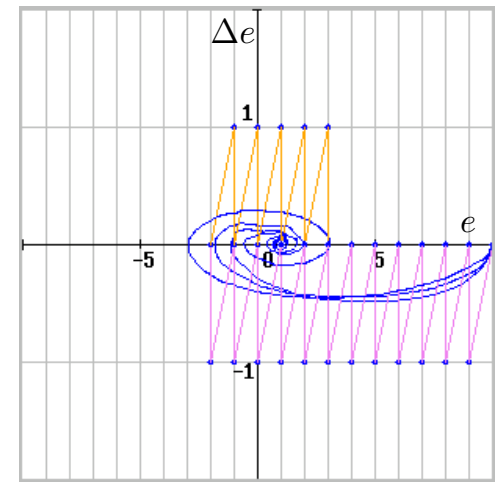

Fig. 7. Phase traces for Exmaple-1 ((i) $C_{1}=8.0$, (ii) $C_{1}=5.0$, (iii) $\left.C_{1}=2.0\right)$.

The input/output characteristic of the discretization process is written by, for example, C-language expression as follows:

$$
\begin{aligned}
& e_{1}^{\dagger}=\gamma *(\text { double })(\text { int })\left(e_{1} / \gamma\right) \\
& v_{1}=0.4 * e_{1}^{\dagger}+3.0 * \text { atan }\left(0.6 * e_{1}^{\dagger}\right) \\
& v_{1}^{\dagger}=\gamma *(\text { double })(\text { int })\left(v_{1} / \gamma\right) .
\end{aligned}
$$

Here, (int) and (double) denote the conversion into an integral number (a round-down discretization) and the reconversion into a double-precision real number, respectively.

When the nominal gain $K=1.0$ and the threshold $\varepsilon_{1}=$ 2.0 are considered, the sectorial area of the point-to-point characteristic for $\varepsilon_{1} \leq\left|e_{1}\right| \leq 40.0$ can be determined as $[0.5,1.5]$.

In this example, the model system is chosen as:

$$
P_{m}(\delta)=\frac{1}{1+C_{1} \delta+8.0 \delta^{2}}, \quad K_{m}=1.0,
$$

and the feedback compensator is chosen as follows:

$$
F(\delta)=\frac{1+C_{1} \delta+8.0 \delta^{2}}{1+8.0 \delta+\delta^{2}} \quad K_{f}=1.0 .
$$

Here, $C_{1}$ is an adjusting parameter.

As for three cases $\left(C_{1}=8.0,5.0,2.0\right)$, the step responses are depicted as shown in Fig. 6, and the phase traces are as shown in Fig. 7. In order to check the robust stability of the discretized control system, $|H|=\left|\Lambda_{1} \Psi_{13} F\right|$ and $\Delta_{1}=$ $1-\beta_{1}\left|\Lambda_{1} \Psi_{13} F\right|$ in (43) for $q_{1}=10.0 \sim 30.0$ are calculated 


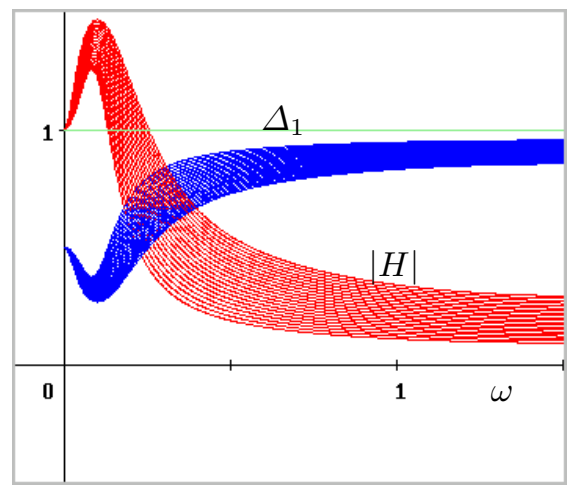

Fig. 8. Checking of robust stability margin for Exmaple-1 when $\beta_{1}=0.5$, $\beta_{2}=\beta_{3}=0, C_{1}=5.0$, and $q_{1}=10.0 \sim 30.0$.

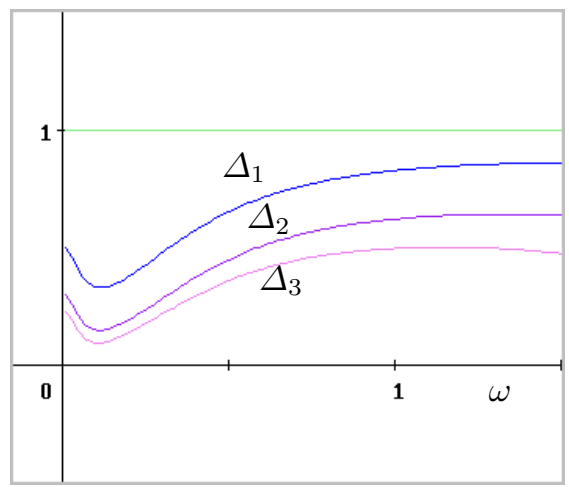

Fig. 9. Checking of robust stability margins for Exmaple-1 when $\beta_{1}=0.5$, $\beta_{2}=0.2, \beta_{3}=0.1$, and $C_{1}=8.0$.

as shown in Fig. 8. Figure 9 shows calculated results of the following principal minors in (36) for $q_{1}=20.0, q_{2}=6.0$, and $q_{3}=3.0$ :

$\Delta_{1}=a_{11}, \quad \Delta_{2}=\left|\begin{array}{ll}a_{11} & a_{12} \\ a_{21} & a_{22}\end{array}\right|, \quad \Delta_{3}=\left|\begin{array}{lll}a_{11} & a_{12} & a_{13} \\ a_{21} & a_{22} & a_{23} \\ a_{31} & a_{32} & a_{33}\end{array}\right|$.

As is obvious from these figures, it can be seen that the stability margins for the discrete control systems are sufficiently proven.

[Example-2] Consider the following model system with time-delay $L_{m}=2.0$ :

$$
P_{m}(\delta)=\frac{1}{1+C_{1} \delta+8.0 \delta^{2}} \mathrm{e}^{-2 s}, \quad K_{m}=1.0 .
$$

The feedback compensator $F(\delta)$ is the same as (54). In this example, the step response and the phase trace become more sufficiently stabilized as shown in Figs. 10 and 11. Figure 12 shows calculated results of the principal minors in (36), $\Delta_{1}, \Delta_{2}$, and $\Delta_{3}$, for $q_{1}=20.0, q_{2}=6.0$, and $q_{3}=3.0$. Obviously, the stability margins for the discrete control systems are sufficiently proven.

\section{CONClusion AND Future REMARKS}

This paper has described a stabilizing and designing problem of discretized model-reference feedback control systems. In particular, a robust stability condition for control systems

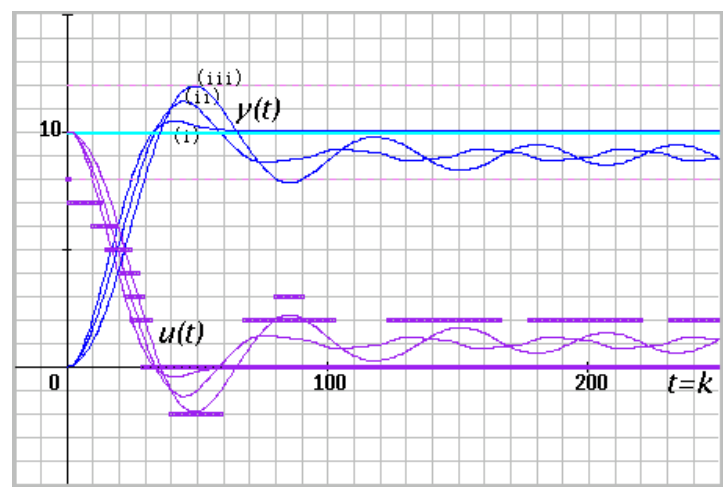

Fig. 10. Step responses for Exmaple-2 ((i) $C_{1}=8.0$, (ii) $C_{1}=5.0$, (iii) $\left.C_{1}=2.0\right)$.

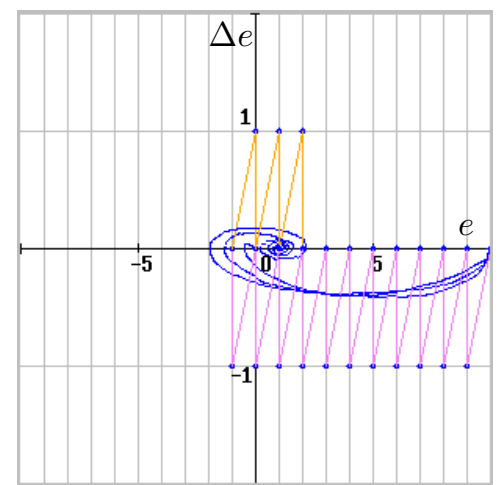

Fig. 11. Phase traces for Exmaple-2 ((i) $C_{1}=8.0$, (ii) $C_{1}=5.0$, (iii) $\left.C_{1}=2.0\right)$.

with multiple discretizations (nonlinearities) was presented in the frequency domain by applying Ostrowski's M-matrix expression. As a consequence, it could be seen that the discretized model-reference control systems are sufficiently stabilized and well designed. The concept described in this paper can be extended to the stabilization of multi-input and multi-output discretized (nonlinear) control systems by using the following general M-matrix representation. (See Appendix.)

\section{APPENDIX}

As was described in the proof of Theorem-1, the following matrix becomes important for the stability of feedback

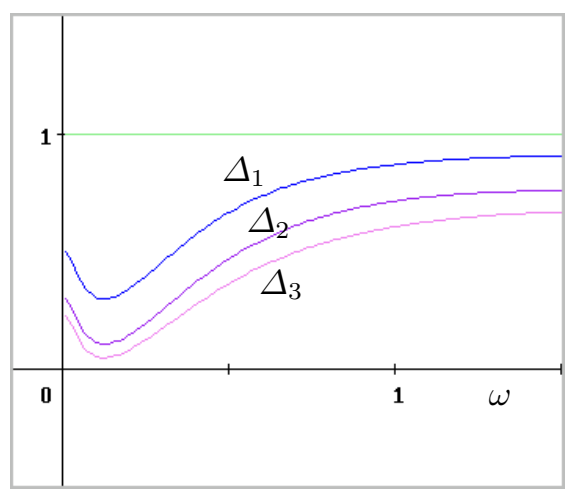

Fig. 12. Checking of robust stability margin for Exmaple- 2 when $\beta_{1}=0.5$, $\beta_{2}=0.2, \beta_{3}=0.1$, and $C_{1}=8.0$. 
systems with multiple nonlinearities:

$$
\mathcal{A}=\left[\begin{array}{cccc}
a_{11} & a_{12} & \ldots & a_{1 n} \\
a_{21} & a_{22} & \ldots & a_{2 n} \\
\vdots & \vdots & \ddots & \vdots \\
a_{n 1} & a_{n 2} & \ldots & a_{n n}
\end{array}\right]
$$

where all non-diagonal elements are non-positive. In addition, if all diagonal elements are positive and if all principal minors of all order are positive, the above matrix $\mathcal{A}$ is called an M-matrix [17].

By using (56), system's inequality can be written as follows:

$$
\left[\begin{array}{cccc}
a_{11} & a_{12} & \ldots & a_{1 n} \\
a_{21} & a_{22} & \ldots & a_{2 n} \\
\vdots & \vdots & \ddots & \vdots \\
a_{n 1} & a_{n 2} & \ldots & a_{n n}
\end{array}\right]\left[\begin{array}{c}
x_{1} \\
x_{2} \\
\vdots \\
x_{n}
\end{array}\right] \leqq\left[\begin{array}{c}
y_{1} \\
y_{2} \\
\vdots \\
y_{n}
\end{array}\right]
$$

Obviously, elements of these vectors in (37) are $x_{i} \geq 0$ and $y_{i} \geq 0$. Moreover, with respect to elements of the matrix $\mathcal{A}$, $a_{i j} \leq 0(i \neq j)$ can be recognized.

The above inequality can be rewritten as follows:

$$
\left[\begin{array}{cccc}
a_{11}^{(1)} & a_{12}^{(1)} & \ldots & a_{1 n}^{(1)} \\
0 & a_{22}^{(2)} & \ldots & a_{2 n}^{(2)} \\
\vdots & \vdots & \ddots & \vdots \\
0 & 0 & \ldots & a_{n n}^{(n)}
\end{array}\right]\left[\begin{array}{c}
x_{1}^{(1)} \\
x_{2}^{(1)} \\
\vdots \\
x_{n}^{(1)}
\end{array}\right] \leqq\left[\begin{array}{c}
y_{1}^{(1)} \\
y_{2}^{(2)} \\
\vdots \\
y_{n}^{(n)}
\end{array}\right]
$$

where

$$
a_{i j}^{(1)}=a_{i j}, \quad x_{j}^{(1)}=x_{j}, \quad y_{i}^{(1)}=y_{i}(i, j=1,2, \cdots, n) .
$$

Furthermore,

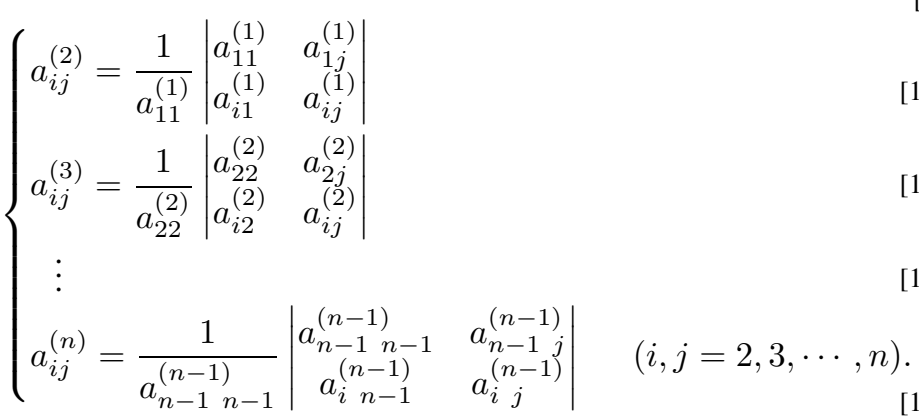

Therefore, the right side of (58) can be written as

$$
\begin{aligned}
& y_{1}^{(1)}=y_{1}, y_{2}^{(2)}=y_{2}^{(1)}-\frac{a_{21}^{(1)}}{a_{11}^{(1)}} y_{1}^{(1)}, y_{3}^{(3)}=y_{3}^{(2)}-\frac{a_{32}^{(2)}}{a_{22}^{(2)}} y_{2}^{(2)} \text {, } \\
& \ldots \ldots, \quad y_{n}^{(n)}=y_{n}^{(n-1)}-\frac{a_{n n-1}^{(n-1)}}{a_{n-1 n-1}^{(n-1)}} y_{n-1}^{(n-1)} \text {, }
\end{aligned}
$$

provided $a_{11}^{(1)}>0, a_{22}^{(2)}>0, \cdots, a_{n-1 n-1}^{(n-1)}>0$. It can be seen that these values are non-negative and bounded if each vector $y_{i}$ is bounded (i.e., $y_{i}^{(1)}<\infty, i=1,2, \cdots, n$ ). In addition, if $a_{n n}^{(n)}>0$ is satisfied, then $x_{n}^{(1)}<\infty, x_{n-1}^{(1)}<$ $\infty, \cdots$, and $x_{1}^{(1)}<\infty$ are obtained in reverse order.
Here, it should be noted that the above conditions $a_{11}^{(1)}>$ $0, a_{22}^{(2)}>0, a_{33}^{(3)}>0, \cdots, a_{n n}^{(n)}$ are rewritten as follows:

$$
\left\{\begin{array}{l}
a_{11}^{(1)}=\Delta_{1}=a_{11}>0 \\
a_{22}^{(2)}=\frac{\Delta_{2}}{\Delta_{1}}=\left|\begin{array}{ll}
a_{11} & a_{12} \\
a_{21} & a_{22}
\end{array}\right| / a_{11}>0 \\
a_{33}^{(3)}=\frac{\Delta_{3}}{\Delta_{2}}=\left|\begin{array}{lll}
a_{11} & a_{12} & a_{13} \\
a_{21} & a_{22} & a_{23} \\
a_{31} & a_{32} & a_{33}
\end{array}\right| /\left|\begin{array}{ll}
a_{11} & a_{12} \\
a_{21} & a_{22}
\end{array}\right|>0 \\
\vdots
\end{array}\right.
$$

The conditions say that all principal minors of matrix $\mathcal{A}$ are positive[18]. That is, it means that the matrix becomes $\mathrm{M}$ matrix.

\section{REFERENCES}

[1] R. E. Kalman, "Nonlinear Aspects of Sampled-Data Control Systems", Proc. of the Symposium on Nonlinear Circuit Analysis, Vol. VI, pages 273-313, 1956.

[2] R. E. Curry, Estimation and Control with Quantized Measurements, Cambridge, MIT Press, 1970.

[3] D. F. Delchamps, "Stabilizing a Linear System with Quantized State Feedback", IEEE Trans. on Automatic Control, Vol. 35, pp. 916-924, 1990.

[4] N. Elia and S. K. Mitter, "Stabilization of Linear System with Limited Information", IEEE Trans. on Automatic Control, Vol. 46, pp. 13841400, 2001.

[5] M. Fu, "Robust Stabilization of Linear Uncertain Systems via Quantized Feedback", Proc. of the IEEE Int. Conf. on Decision and Control, TuA06-5, 2003

[6] C. A. Desoer and M. Vidyasagar, Feedback System: Input-Output Properties, Academic Press, 1975.

[7] C. J. Harris and M. E. Valenca, The Stability of Input-Output Dynamical Systems, Academic Press, 1983.

[8] Y. Okuyama, "Robust Stability Analysis for Discretized Nonlinear Control Systems in a Global Sense", Proc. of the 2006 American Control Conference, Minneapolis, USA, pp. 2321-2326, 2006.

[9] Y. Okuyama. "Discretized PID Control and Robust Stabilization for Continuous Plants", Proc. of the 17th IFAC World Congress, Seoul, Korea, pp. 1492-1498, 2008.

[10] Y. Okuyama, "Discretized PID Control and Robust Stabilization for Continuous Plants on an Integer-Grid Pattern", Proc. of the European Control Conference, Budapest, Hungary, pp. 514-519, 2009.

[11] Y. Okuyama, "Model-Reference Discretized PID Control and Robust Stabilization for Continuous Plants", Proc. of the 18th IFAC World Congress, Milano, Italy, pp. 5813-5818, 2011.

[12] Y. Okuyama, "Discretized Model-Reference Feedback and Robust Stabilization on Integer Grid Coordinates", Proc. of the 7th IFAC Symp. on Robust Control Design, Aalborg, Denmark, pages 400-405, 2012.

[13] Y. Okuyama, "Estimation of Parameter Sensitivity of Linear Control Systems and Synthesis of Passive Adaptive Control Systems", Journal of SICE, Vol. 3, pages 759-767, 1964 (in Japanese).

[14] F. Takemori and Y. Okuyama, "Discrete-Time Model Reference Feedback and PID Control for Interval Plants", Proc. of the IFAC Workshop on Digital Control, Terrassa, Spain, pp. 260-265, 2000.

[15] Y. Okuyama et al., "Robust Stability Evaluation for Sampled-Data Control Systems with a Sector Nonlinearity in a Gain-Phase Plane", Int. J. of Robust and Nonlinear Control, volume 9, No. 1, pp. 15-32, 1999.

[16] Y. Okuyama et al., "Robust Stability Analysis for Non-Linear Sampled-Data Control Systems in a Frequency Domain", European Journal of Control, Vol. 8, No. 2, pp. 99-108, 2002.

[17] A. M. Ostrowski, "Note on Bounds for Some Determinants", Duke Mathematical Journal, Vol. 22, No.1, pp. 95-102, 1955.

[18] Y. Okuyama, "On the $L_{2}$-Stability of Linear Systems with TimeVarying Parameters", Transaction of SICE, Vol. 3, pages 252-259, 1967 (in Japanese). 